\title{
DERMOSCOPY AS A VALUABLE TOOL IN DIAGNOSIS OF NODULAR AMELANOTIC MELANOMA AND NODULAR BASAL CELL CARCINOMA
}

\author{
Smolarova M, Minarikova E. \\ Dermatovenerology Clinic, Jessenius Faculty of Medicine in Martin, Comenius University in \\ Bratislava and University Hospital Martin, Slovakia
}

\begin{abstract}
Nodular amelanotic melanoma has been always a great challenge in dermatology. Because of lack of melanin pigment, tumors are diagnosed usually in advanced stage. Amelanotic melanoma can mimic basal cell carcinoma. Knowledge of typical dermoscopic structures helps to establish diagnosis and to plan surgery with appropriate safety margins. In amelanotic melanoma we can see typical vessels, white streaks or milky red globules on pink-reddish background. Vessels are typically thin and polymorphous in thick amelanotic melanoma. We had a case when vessels were polymorphous but thick. It can be confusing with nodular basal cell carcinoma where vessels are typically thick and arborizing. Nodular basal cell carcinoma is the most common form of basal cell carcinoma. Dermoscopy is a valuable tool for the diagnosis of basal cell carcinoma. Typical dermoscopic structures are arborizing vessels, possible sites of ulceration and/or pigmentation. We describe a case report of patient with typical dermoscopic structures seen in nodular basal cell carcinoma.
\end{abstract}

Key words: Dermoscopy, nodular amelanotic melanoma, basal cell carcinoma,

\section{INTRODUCTION}

Nodular amelanotic melanoma represents $1.8-8.1 \%$ of all melanomas /3/. Nodular amelanotic melanoma has been always a great challenge in dermatology. Because of lack of melanin pigment, tumors are diagnosed usually in advanced stage. In fact, amelanotic melanoma is a great masquerader that can mimic a range of skin lesions including inflammatory skin lesions and also benign tumors such as hemangioma, pyogenic granuloma, lichen planus-like keratosis or intradermal nevus that are not routinely excised /8/. Amelanotic melanoma can mimic basal cell carcinoma. Knowledge of typical dermoscopic structures helps to establish diagnosis and to plan surgery with appropriate safety margins. In amelanotic melanoma we can see typical vessels, white strikes or milky red globules on pink-reddish background. Vessels are typically thin and polymorphous in thick amelanotic melanoma. We had a case when vessels were polymorphous but thick. It can be confusing with nodular basal cell carcinoma where vessels are typically thick and arborizing.

This tumor presents often as pink to red symmetric nodule with well-defined borders and thus escape the clinical ABCD criteria (Fig. 1). For this reason, the EFG rule has been introduced to diagnose nodular melanoma subtypes. Thereby, E stands for elevation, $\mathrm{F}$ for firm on palpation, and $\mathrm{G}$ for continuous growth for more than 1 month $/ 4 /$.

Address for correspondence:

Marketa Smolarova, MD, Dermatology Outpatient Clinic, Prieloztek 1, 03601 Martin, Slovakia phone: +421 0905 647233, e-mail.: smolarovam@gmail.com 


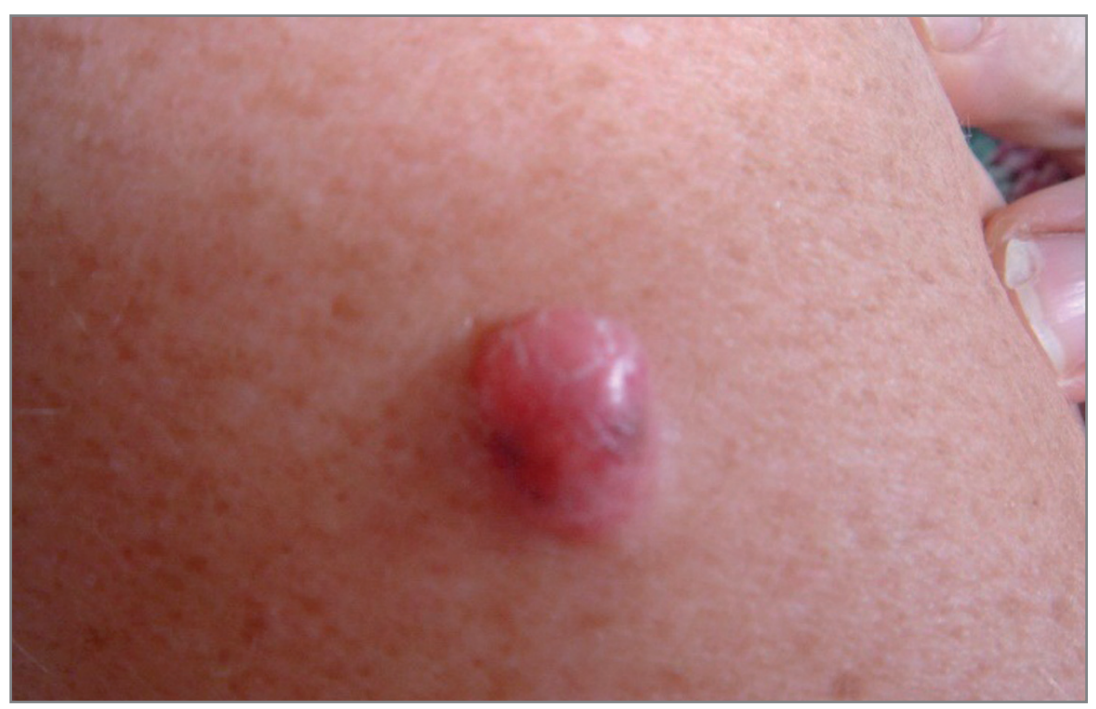

Fig. 1 Clinical picture

of amelanotic melanoma

Given the lack of pigmentation, the only clue for the dermoscopic diagnosis of amelanotic melanoma are vessels. The vascular morphology strongly depends on tumor thickness and, subsequently, the vascular patterns undergo time-related changes during melanoma progression.

Early amelanotic melanoma in situ shows regularly arranged dotted vessels over a pink background. At times, a white network (reticular depigmentation) may be present/9/. Early invasive amelanotic melanoma (less than $1 \mathrm{~mm}$ thickness) typically exhibits a combination of dotted and small coiled vessels. A further dermoscopic clue when using polarized dermoscopy is represented by presence of short white streaks.

Intermediate thick melanoma (1-2 $\mathrm{mm})$ is typified by polymorphous vessels consisting of dotted, coiled, and linear, irregular vessels over a pink background. The linear vessels are most commonly located in the center (most invasive part), whereas the dotted vessels are typically seen in periphery.

Thick melanoma (more than $2 \mathrm{~mm}$ ) often appears clinically as an EFG-positive nodule. Dermoscopy exhibit polymorphous atypical vessels consisting of kinked linear, hairpin and large coiled vessels. Typically, these vessels are of small caliber and appear on a reddish background. A further criterion that can be present is milky red globules which give rise to papillomatous tumoral surface. In addition, ulceration may be found / 10/.

Basal cell carcinoma is a common human skin malignancy, which rarely metastasizes but has the potential to cause problematic local tissue invasion. Currently, despite the description of more than 26 different subtypes of basal cell carcinoma, a universally accepted classification scheme is lacking/ $1 /$. There are several main subtypes of BCC: nodular, superficial, morpheaform, fibroepithelioma of Pinkus and basosquamous carcinoma. Some of them can be pigmented. Combinations of each types may occur.

Patient history and clinical examination are very important first steps in reaching a correct diagnosis. Dermoscopy is also a valuable tool for the diagnosis basal cell carcinoma. The dermoscopic diagnosis relies on the evaluation of blood vessel morphology and architecture, as ulceration and possible areas of pigmentation /10/.

Nodular basal cell carcinoma is the most common form of BCC (Fig .2). The lesion begins as a pearly white or pink dome-shaped papule or nodule. Vessels become prominent and easily recognizable through the thin epidermis as the lesion enlarges. The center frequently ulcerates and bleeds and subsequently accumulates crust and scale. Ulcerated BCCs were formerly termed rodent ulcers. Ulcerated areas heal with scarring, and patients often 
assume their conditions are improving. This cycle of growth, ulceration, and healing continues as the mass extends peripherally and deeper /3/. Nodular BCC often occurs on head and neck sites/6/. Clinical differential diagnoses include both benign and malignant lesions, such as dermal nevus (particularly Miescher nevi of the face), epidermoid cyst, sebaceous hyperplasia, seborrheic keratosis, squamous cell carcinoma and amelanotic/hypomelanotic melanoma $/ 2 /$.

Fig.2 Clinical picture of basal cell carcinoma

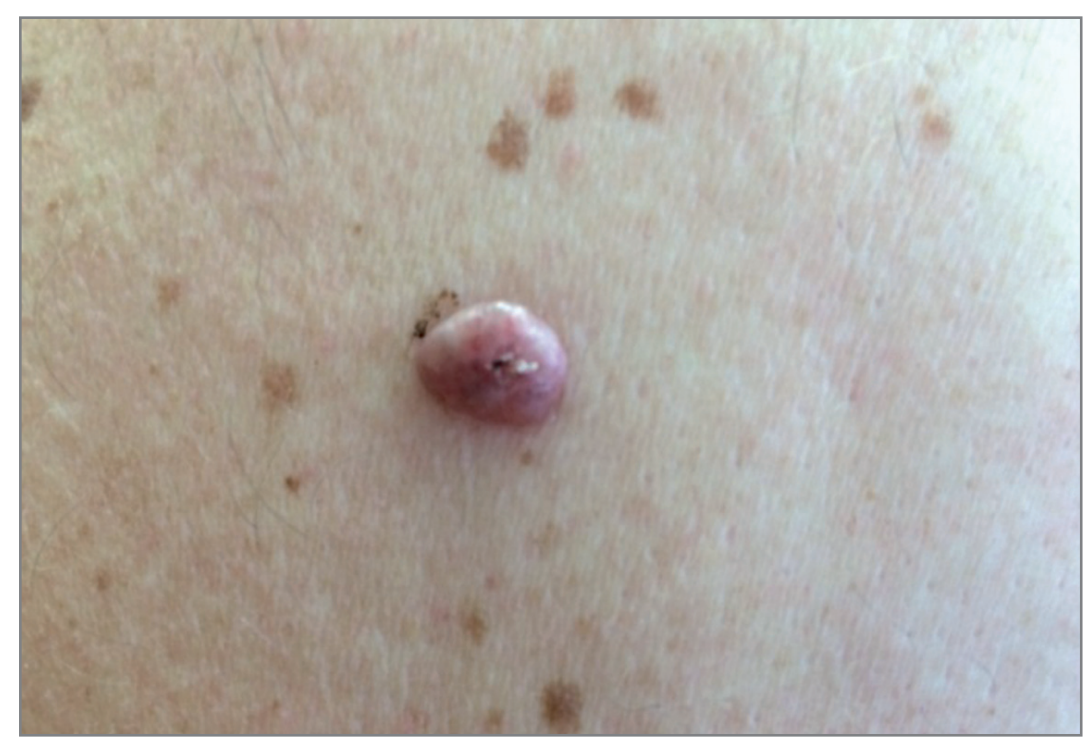

Dermoscopicaly, non-pigmented nodular BCC is characterized by tree-like arborizing telengiectasias, which usually arise as bright red, large diameter stem vessels dividing into finer, sharply focused ramifications over the surface of the lesion. The vessels generally occur on a homogenous white-pinkish background, but white streaks (lines) may also be seen under polarized light /5/. Orange-to-red, reddish-brown, or reddish-black ulceration and an erythematous blush may also be seen. Pigmentation may present as blue-gray globules, blue-gray ovoid nests, spoke wheel-like and leaf-like areas.

The management of nodular BCC includes surgical excision or curettage with electro-desiccation/10/.

\section{METHODS}

We investigate lesion clinically first. Inspection describes colors and possible ulceration. Palpation is necessary for finding soft or firm consistency. We measure size of lesion. There is important history of duration, elevation, possible bleeding or oozing, itching, burning, pain or other sensation of lesion. Dermoscopy enables visualization of colors and structures in epidermis and papillary dermis. These structures are invisible with naked eyes. We use non-contact hand held dermoscop Derm-lite (fig. 3) with polarized light and magnification 10x for first dermoscopic investigation. We continue with more precious and detailed visualization of lesion with digital dermoscop Derm DOC 2.3. (fig. 4) This device is equipped with polarized light and can magnify lesion till 100x. It is excellent for observation of vessels and other delicate dermoscopic structures. There is also possibility of saving lesion in memory of computer which is attached to digital dermoscop and enables comparing or consultation later. 


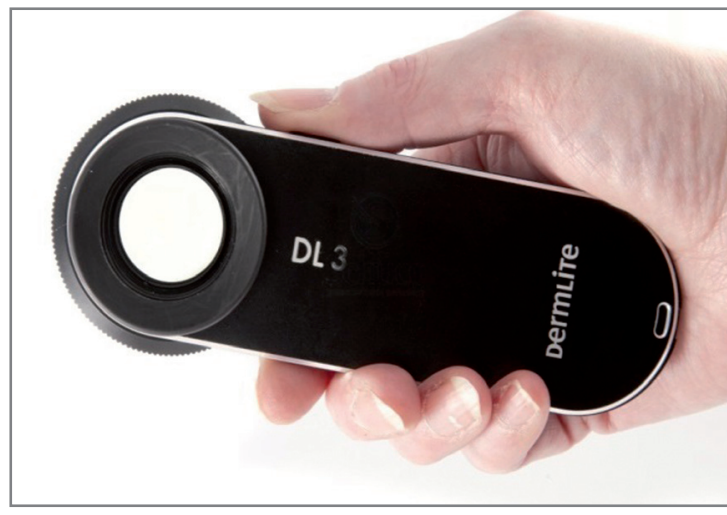

Fig. 3 Hand held dermoscop Derm-lite

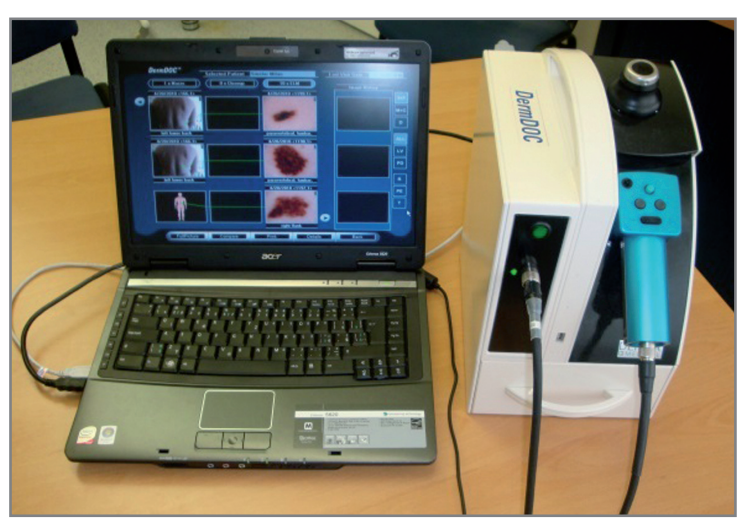

Fig. 4 Digital dermoscop Derm DOC 2.3.

\section{Case report 1}

We had a 45 years old female patient, having a reddish nodule 10x9 $\mathrm{mm}$ growing on her right arm for approximately 1 year. The last 6 months she noted that lesion is more elevated. The nodule was firm on palpation. There was no sensation as a pain, itching or burning. Borders of nodule were well defined. There was a little amount of brown pigmentation at the bottom. The surface of lesion was without an ulceration.

We saw vessels and white streaks on the surface of lesion with handheld dermoscop and small amount of brown pigmented globules at the bottom. A polarized dermoscopy with magnification 30x showed small islets of homogenous pigment, couple of asymmetric brown globules at the bottom, white streaks and linear, curved, serpentine and even lightly branching vessels of different calibers (Fig. 5).

A magnification 60x shows better thick linear vessels, thin polymorphous atypical vessels, white streaks and asymmetric brown globules on pink-reddish background (Fig. 6).

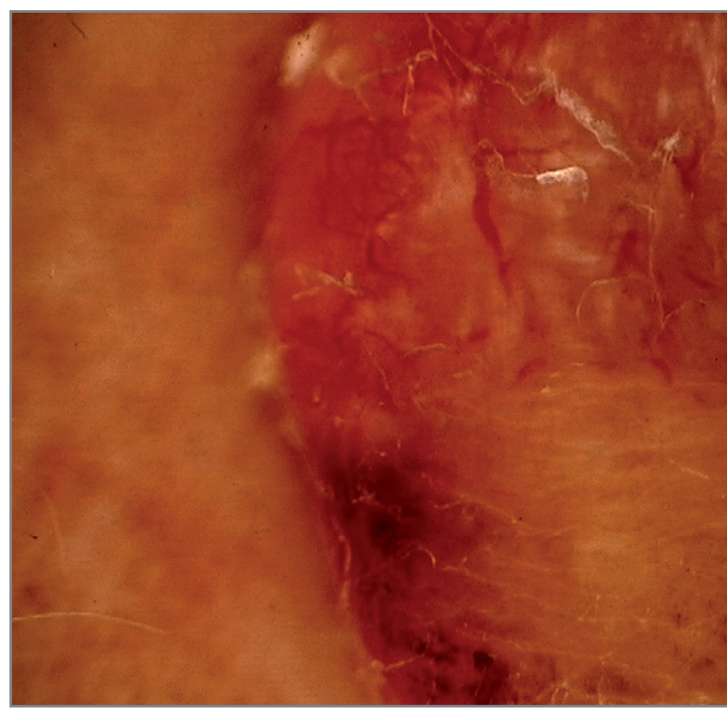

Fig. 5 Amelanotic melanoma Mag. 30x thick linear vessels, thin polymorphous atypical vessels, white streaks, brown globules at the bottom

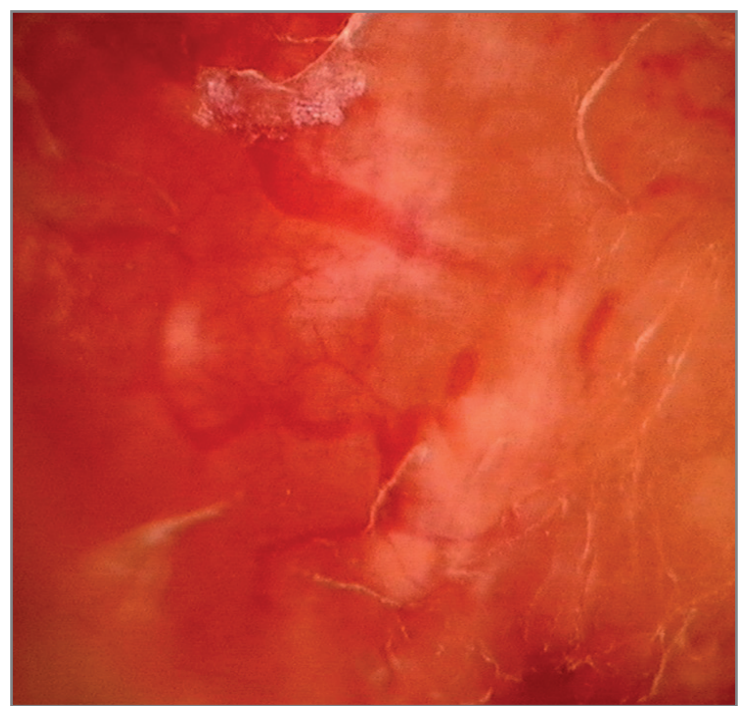

Fig. 6 Amelanotic melanoma Mag.60x thick linear vessels, thin polymorphous atypical vessels, white streaks 
There was a strong suspicion for hypomelanotic or amelanotic melanoma. A possibility of nodular basal cell carcinoma was in differential diagnosis.We suggested to remove tumor with $2 \mathrm{~cm}$ safety margins.

Histology: Nodular amelanotic melanoma without ulceration (Fig. 7). Growing phase vertical, Breslow: 9,6 mm. Clark V, Mitotic activity index: 6-8mf /mm2, Melan A+, HMB45+, S100mo+, Vimentin+, Melanin pigment -, Infiltration with lymphocytes+, TNM: pT4A pNx pMx.

Fig. 7 Amelanotic melanoma, histopathologic picture. Pleomorphic cells and lack of melanin pigment (HE, obj. 40x)

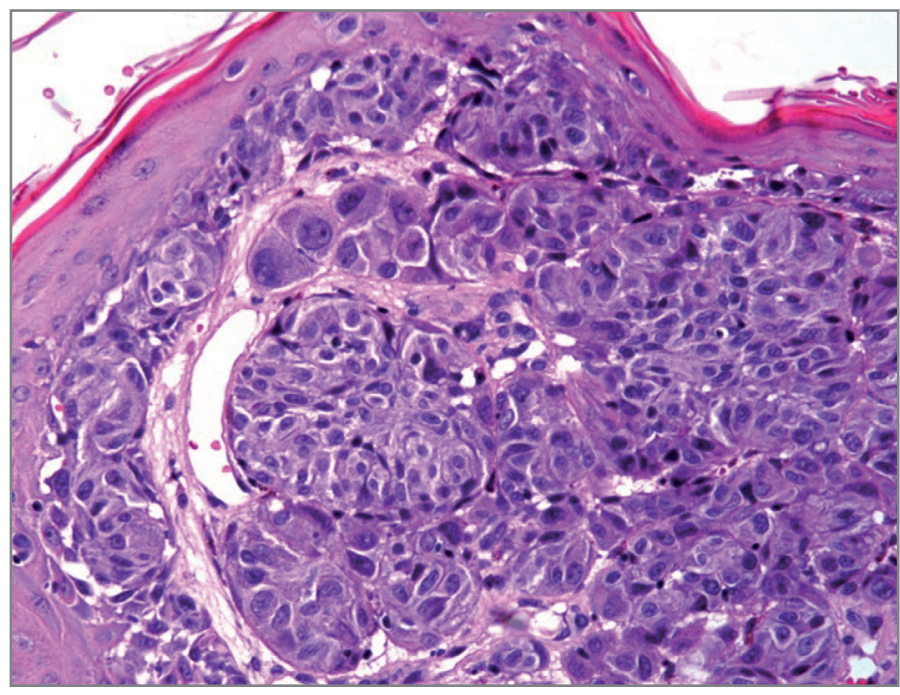

\section{Case report 2}

We had a 65-year-old male patient with slowly growing firm nodule lasting approximately 5 years on his right arm. Clinically the lesion was pinkish-red dome-shaped nodule with diame-

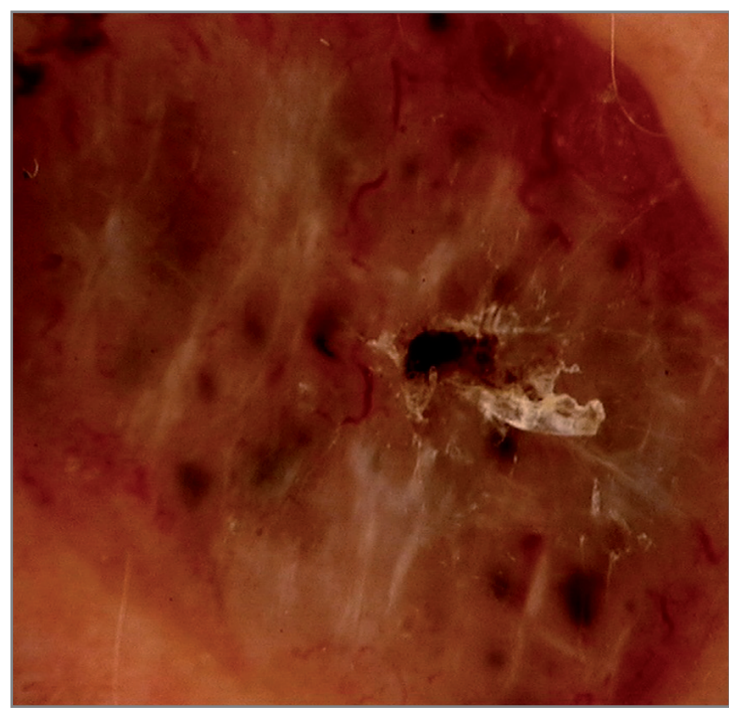

Fig. 8 Basal cell carcinoma Mag. 30x Multiple blue-gray ovoid and globular structures, white streaks, ulceration

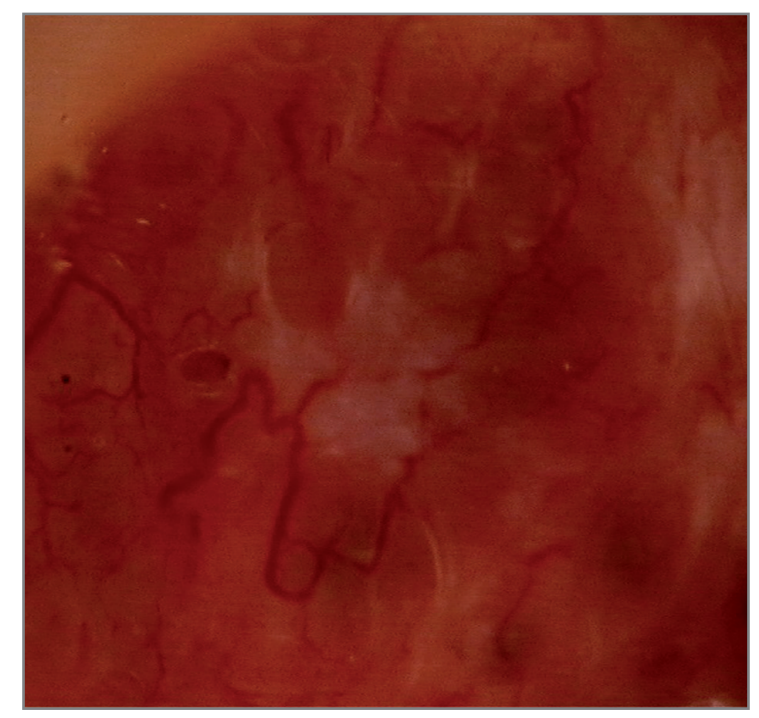

Fig. 9 Basal cell carcinoma Mag.60x red-bright arborizing vessels 
ter of 9x10 mm. The nodule was firm, there was little ulceration and red-brown crust. Patient noticed infrequent bleeding and subsequent healing in the past. There was no pain or other sensation.

Investigation with digital dermatoscop with magnification 30x and 60x revealed typical dermoscopic structures.Dermoscopicaly we saw pinkish-white background with prominent thick red-bright arborizing vessels, white streaks, multiple blue-gray ovoid and globular structures. There was also small area of microulceration in the center, covered with red-brown crust and white scales (Fig. 8, 9). It is necessary not to press with dermatoscop on lesion, otherwise the vessels disappear. The lesion showed typical signs of basal cell carcinoma. We suggested surgical excision with $5 \mathrm{~mm}$ safety margins.

Histopathology report: Nodular basal cell carcinoma, solid type with cystic degeneration, infiltrating dermis 3,8 mm. Free resection margins (Fig. 10).

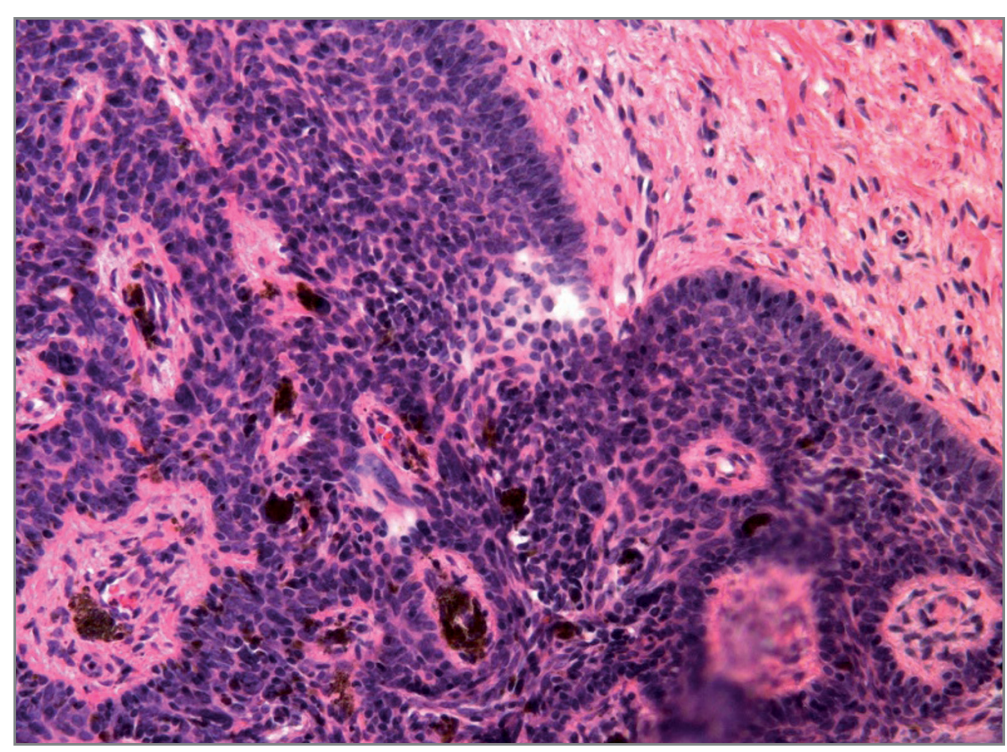

Fig.10 Pigmented nodular basal cell carcinoma (HE, obj. 40x)

\section{DISCUSSION}

Although the linear vessels of thick melanoma are commonly small, large and thick vessels do not exclude melanoma $/ 7 /$. We had a case when vessels were mostly thick linear and thin polymorphous. It can be confusing with nodular basal cell carcinoma where vessels are typically thick and arborizing. It is important not to press with dermoscop too much on lesion, otherwise vessels disappear. The presence of white streaks and brown globules is also frequently found in basal cell carcinoma. Nodular basal cell carcinoma is the most common form of BCC. Clinical and dermoscopic differential diagnoses include both benign and malignant lesions, such as dermal nevus (particularly Miescher nevi of the face), epidermoid cyst, sebaceous hyperplasia, seborrheic keratosis, squamous cell carcinoma and amelanotic/hypomelanotic melanoma $/ 2 /$. The main clue can be recognition of the vessel patterns. Arborizing vessels are typical for basal cell carcinoma, hairpin pattern is typical for irritated seborrheic keratosis, crown pattern is typical for sebaceous hyperplasia. Polymorphous pattern is found in amelanotic or hypomelanotic melanoma and squamous cell carcinoma. 


\section{CONCLUSIONS}

Dermoscopy of thick amelanotic melanoma can present with thick and thin polymorphous atypical vessels, which can be linear, curved, serpentine or lightly branching. We can observe also white streaks in polarized dermoscopy. These dermoscopic structures should raise a red flag of concern that lesion can be malignant and we should solve the problem immediately and properly. We recommended extirpation of sentinel lymph node in right axilla, $\mathrm{X}$ ray of chest and ultrasonography of abdomen. In general, sentinel lymph node biopsy is recommended for primary melanomas more than 1,0 mm. Sentinel lymph node biopsy was negative. Regular checkup at Skin Cancer Clinic of the University Hospital in Martin was set for every 3 months. Interferon can be given as high dose interferon for one year for improvement in distant metastasis-free survival.

Dermoscopy of nodular basal cell carcinoma can present with pinkish-white background with prominent thick red-bright arborizing vessels, white lines, multiple blue-gray ovoid and globular structures, microulceration covered with red-brown crust and white scales. These dermoscopic structures should raise a suspicion that lesion can be malignant and we should solve the problem correctly. We suggested regular follow-up every 6 months for possible recurrence of basal cell carcinoma. We check the scar and observe the skin on the whole body to find possible new skin cancer. We also recommend a photoprotection to prevent other sun damage on the skin.

\section{REFERENCES}

1. Bolognia JL, Jorizzo LJ, Schaeffer JV, Dermatology $3^{\text {rd }}$ edition, Elsevier, 2012: 1784

2. Cockerell CJ, Tran KT, Carucci j et al. Basal cell carcinoma. In: Rigel DS, Robinson Jk, Ross M et al. (eds). Cancer of the Skin. Philadelphia, PA: Elsevier-Saunders, 201 1,2nd ed.: 99-123.

3. Habif T. Clinical Dermatology. Elsevier 6th ed.2016: 812, 874

4. Kelly JW, Chamberlain AJ, Staples MP, McAvoy B. Nodular melanoma. No longer as simple as ABC. Aust Fam Physician 2003,32(9): 706-9.

5. Liebman TN, Jaimes-Lopez N, Balagula Y et al. Dermoscopic features of basal cell carcinomas: differences in appearance under non-polarized and polarized light. DERMATOL Surg 2012, 38: 392-9.

6. McCormack CJ, Kelly JW, Dorevitch AP. Differences in age and body site distribution of the histological subtype of basal cell carcinoma: A possible indicator of differing causes. Arch dermatol 1997, 133: 593-6

7. Menzies SW, Moloney FJ, Byth K et al. Dermoscopic evaluation of nodular melanoma. JAMA Dermatol 2013; 149(6): 699-709

8. Puig S, Argenziano G, Zalaudek I et al. Melanomas that failed dermoscopic detection. A combined clinicodermoscopic approach for not missing melanoma. Dermatol Surg 2007,33(10): 1262-73

9. Zalaudek I, Kreusch J, Giacomel J, Ferrara G, Catricala C, Argenziano G. How to diagnose non -pigmented skin tumors: A review of vascular structures seen with dermoscopy: Part I. Melanocytic skin tumors. J Am Acad Dermatol 2010, 63(3): 361-74

10. Zalaudek I, Argenziano G, Giacomel J. Dermatoscopy of Non-pigmented skin Tumors: Pink-thinkblink. CRC Press, 2016: 66, 93

Received: February, 2, 2017

Accepted: March, 24, 2017 